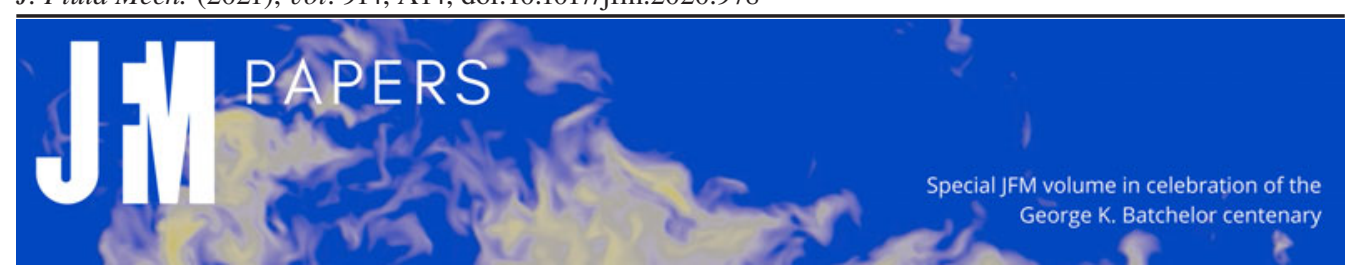

\title{
Multi-scale steady solution for Rayleigh-Bénard convection
}

\author{
Shingo Motoki ${ }^{1} \uparrow$, Genta Kawahara ${ }^{1}$ and Masaki Shimizu ${ }^{1}$ \\ ${ }^{1}$ Graduate School of Engineering Science, Osaka University, 1-3 Machikaneyama, Toyonaka, \\ Osaka 560-8531, Japan
}

(Received 14 April 2020; revised 17 September 2020; accepted 2 November 2020)

We found a multi-scale steady solution of the Boussinesq equations for Rayleigh-Bénard convection in a three-dimensional periodic domain between horizontal plates with a constant temperature difference. This was realised using a homotopy from the wall-to-wall optimal transport solution provided by Motoki et al. (J. Fluid Mech., vol. 851, 2018, R4). A connected steady solution, which is a consequence of bifurcation from a thermal conduction state at Rayleigh number $R a \sim 10^{3}$, is tracked up to $R a \sim 10^{7}$ using a Newton-Krylov iteration. The three-dimensional exact coherent thermal convection exhibits a scaling of $N u \sim R a^{0.31}$ (where $N u$ is the Nusselt number) as well as multi-scale thermal plume and vortex structures, which are quite similar to those in turbulent Rayleigh-Bénard convection. The mean temperature profiles and the root-mean-square of the temperature and velocity fluctuations are in good agreement with those of the turbulent states. Furthermore, the energy spectrum follows Kolmogorov's $-5 / 3$ scaling law with a consistent prefactor, and the energy transfer to small scales with a nearly constant flux in the wavenumber space is in accordance with the turbulent energy transfer.

Key words: Bénard convection, turbulent convection

\section{Introduction}

Rayleigh-Bénard convection, a buoyancy-driven flow in a horizontal fluid layer heated from below and cooled from above, is one of the most canonical flows widely observed in nature, and also in engineering applications. The effect of buoyancy on a flow is characterised by the Rayleigh number $R a$. When $R a$ exceeds a certain critical value $R a_{c}$, the thermal conduction state becomes unstable and two-dimensional (2-D) steady convection rolls appear (Drazin \& Reid 1981). At a higher $R a$, the convection becomes

$†$ Email address for correspondence: motoki@me.es.osaka-u.ac.jp

(C) The Author(s), 2021. Published by Cambridge University Press. This is an Open Access article, distributed under the terms of the Creative Commons Attribution licence (http://creativecommons.org/ licenses/by/4.0/), which permits unrestricted re-use, distribution, and reproduction in any medium, provided the original work is properly cited. 


\section{S. Motoki, G. Kawahara and M. Shimizu}

time-dependent and subsequently exhibits turbulent states with multi-scale thermal and vortex structures. One of the primary interests in the Rayleigh-Bénard problem is the scaling of turbulent heat transfer with $R a$, i.e. the dependence of the Nusselt number $N u$ on $R a$. Over half a century ago, Priestley (1954) and Malkus (1954) simultaneously and independently proposed $N u \sim R a^{1 / 3}$. Priestley derived the scaling from a similarity argument based on dimensional analysis by assuming that the heat flux is independent of the height of the fluid layer, $H$, while leaving the Prandtl number $P r$ dependence unspecified. In contrast, Malkus obtained a scaling independent of $\operatorname{Pr}$ based on a marginal stability argument. Later Malkus' theory was reframed and developed by Howard (1966). Malkus (1954) also raised a question for the upper limit of the heat transfer in thermal convection, and the upper bounds of $N u \sim R a^{1 / 2}$ were derived via variational approaches (Howard 1963; Busse 1969). In the 1990s, Doering \& Constantin (1996) derived a rigorous upper bound of $N u \leq 0.167 R a^{1 / 2}-1$ by employing a new variational approach called 'the background method' (Doering \& Constantin 1992), and then the lowest asymptotic upper bound, $N u-1=0.02634 R a^{1 / 2}$, was obtained by Plasting \& Kerswell (2003). Based on the mixing-length theory, apart from the upper-bound problems, a scaling of $N u \sim$ $\operatorname{Pr}^{1 / 2} \mathrm{Ra}^{1 / 2}$ was derived by Spiegel (1963). In 1961, his theory was already proposed as testified by Batchelor (1961) (see also Doering 2019). Shortly afterwards, Kraichnan (1962) modified Spiegel's theory, and predicted $N u \sim \operatorname{Pr}^{1 / 2} R a^{1 / 2}(\ln R a)^{-3 / 2}$ for a sufficiently large $R a$, as a scaling in an asymptotic state with turbulent boundary layers. The logarithmic correction possibly varies from $(\ln R a)^{-3 / 2}$ to $(\ln R a)^{-3}$ (Chavanne et al. 1997); $N u \sim \operatorname{Pr}^{1 / 2} \mathrm{Ra}^{1 / 2}$ is currently called 'ultimate' scaling. In conventional turbulent Rayleigh-Bénard convection, however, the ultimate scaling has not been observed yet. A prominent experiment by Niemela et al. (2000) for a very high $R a$ exhibits $N u \sim R a^{0.31}$ even at $R a \sim 10^{17}$. A recent numerical simulation by Iyer et al. (2020) up to $R a=10^{15}$, in a slender cylindrical container with an aspect ratio of $1 / 10$, exhibits $N u \sim R a^{0.33}$. In the 1990s, Shraiman \& Siggia (1990) deduced $\mathrm{Nu} \sim \mathrm{Pr}^{-1 / 7} \mathrm{Ra}^{2 / 7}$ by assuming the existence of turbulent boundary layers. In the 2000s, Grossmann \& Lohse (2000, 2001, 2002, 2004) proposed a unifying scaling law of global properties for $\mathrm{Ra}$ and $\mathrm{Pr}$, based on decomposing the total scalar and energy dissipation into contributions from the bulk region and the boundary layer. Their scaling argument results in two equations for the Nusselt number $N u(R a, P r)$ and the Reynolds number $\operatorname{Re}(R a, P r)$ with six adjustable parameters, and they are determined to fit experimental and numerical data. Many experiments and numerical simulations have demonstrated the validity of this scaling law (Ahlers, Grossmann \& Lohse 2009; Stevens et al. 2013). Per the argument, the scaling $N u \sim R a^{1 / 3}$ is derived in the high- $R a$ regime $10^{8} \lesssim R a \lesssim 10^{14}$ for $\operatorname{Pr} \sim 1$. The transition to the ultimate scaling is also predicted for $R a \gtrsim 10^{14}$; however, the local effective behaviour is represented by $N u \approx c R a^{0.38}$, where $c$ is a fitting constant, due to logarithmic corrections (Grossmann \& Lohse 2011). Although some results have shown the transition to $N u \sim R a^{0.38}$, the high- $R a$ scaling is still being discussed (Chillà \& Schumacher 2012; Zhu et al. 2018; Doering, Toppaladoddi \& Wettlaufer $2019 a, b)$. In contrast, for $10^{8} \lesssim R a \lesssim 10^{11}$, a considerable amount of turbulent data exhibit the classical scaling $\widetilde{N u} \sim \mathrm{Ra}^{1 / 3}$ (e.g. Niemela \& Sreenivasan 2006; He et al. 2012).

Recently, Waleffe, Boonkasame \& Smith (2015) found a scaling of $N u=0.115 R a^{0.31}$ in 2-D steady Rayleigh-Bénard convection for $10^{7} \lesssim R a \lesssim 10^{9}$ and $\operatorname{Pr}=7$ (see also Sondak, Smith \& Waleffe 2015). In their work, 2-D steady solutions were obtained to maximise $N u$ by optimising the horizontal periods. The scaling is achieved by a family of $2-\mathrm{D}$ solutions with a horizontal period that decreases with increasing $R a$. Interestingly, the scaling is quite similar to the three-dimensional (3-D) turbulent data fit $N u=0.105 R a^{0.312}$ 


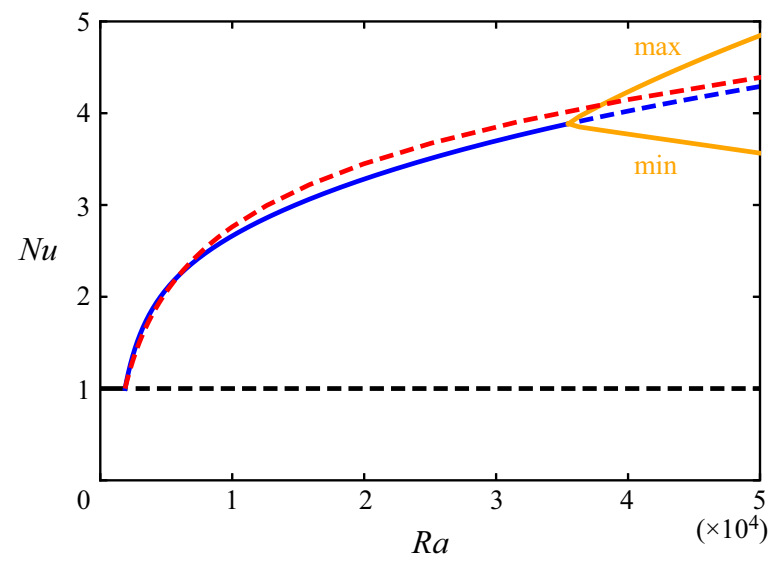

Figure 1. Nusselt number $\mathrm{Nu}$ as a function of Rayleigh number $\mathrm{Ra}$. The red and blue curves represent the 3-D steady solution in the square periodic domain and 2-D steady solution with $L / H=\pi / 2$ for $\operatorname{Pr}=1$ bifurcating from the conduction state (black) at $R a \approx 1879$, respectively. The orange curves show the maximum and minimum values of $\mathrm{Nu}$ in the 3-D time-periodic solution. The dashed lines denote unstable solutions.

(He et al. 2012). Although the result suggests that simple and coherent structures can capture the essence of convective turbulence, it does not imply that this can be realised by any single 2-D steady solution with a fixed horizontal period (fundamental wavelength). More recently, a wall-to-wall optimal transport problem, which is a variational problem for finding a divergence-free velocity field optimising scalar transport between two parallel plates, has been discussed (Hassanzadeh, Chini \& Doering 2014; Tobasco \& Doering 2017; Doering \& Tobasco 2019). Motoki, Kawahara \& Shimizu (2018a) also numerically obtained 3-D time-independent velocity fields to be the optimal states maximising heat transfer between two isothermal no-slip parallel plates under the constraint of fixed total enstrophy. The optimal states exhibit ultimate scaling, which is quite close to the lowest rigorous upper bound obtained by Plasting \& Kerswell (2003), and lead to hierarchical self-similar vortex structures. In the optimised 3-D velocity fields, smaller and stronger vortices appear closer to the walls as the total enstrophy increases. In velocity fields numerically optimised within a 2-D field, the emergence of such hierarchical flow structures has not been observed (Souza, Tobasco \& Doering 2020). Although the 3-D optimal state needs an external body force other than buoyancy, we proved that the optimal state found by Motoki et al. (2018a) could continuously connect to a steady solution of the Boussinesq equations using the homotopy continuation method (see appendix A).

In this paper, we discuss the connected 3-D steady solution. In the Rayleigh-Bénard convection between horizontal boundaries, a 3-D steady solution with convection cells also bifurcates from the conduction state at the same critical value of $R a$ as the 2-D steady solution (see figure 1). This is because convection rolls in any horizontal direction can exist simultaneously. Although the 3-D solution is not stable, it exists even at a high $R a$. We demonstrate the ability of this invariant solution to capture key statistical features, as well as coherent thermal and flow structures in turbulent Rayleigh-Bénard convection. We then discuss the hierarchical multi-scale vortex structures and energy transfer in the 3-D steady solution.

The remainder of the paper is organised as follows. In $\S 2$, we introduce the governing equations, boundary conditions and dimensionless parameters to characterise the thermal convection. We then describe the numerical procedures to obtain nonlinear solutions. 


\section{S. Motoki, G. Kawahara and M. Shimizu}

The statistical properties and spatial structures of the 3-D steady solution are presented in $\S 3$, and the hierarchical vortex structures are discussed in $\S 4$. Finally, a summary and conclusions are presented in $\S 5$. In appendix A, we present the homotopy continuation analysis from the 3-D optimal solution of the Euler-Lagrange equations for the wall-to-wall optimal transport problem to the present 3-D steady solution of the Boussinesq equations. The parameter dependence of the 3-D steady solution and the adequacy of the spatial resolution are discussed in appendix B.

\section{Boussinesq equations and numerical methods}

Let us consider a fluid layer between two horizontal plates heated from below and cooled from above. We employ the Oberbeck-Boussinesq approximation, wherein the density variations are only significant in the buoyancy term. The time evolutions of velocity field $\boldsymbol{u}(\boldsymbol{x}, t)=u \boldsymbol{e}_{x}+v \boldsymbol{e}_{y}+w \boldsymbol{e}_{z}$ and temperature field $T(\boldsymbol{x}, t)$ are described by the Boussinesq equations

$$
\begin{gathered}
\nabla \cdot \boldsymbol{u}=0 \\
\frac{\partial \boldsymbol{u}}{\partial t}+(\boldsymbol{u} \cdot \nabla) \boldsymbol{u}=-\frac{1}{\rho} \nabla p+v \nabla^{2} \boldsymbol{u}+g \alpha T \boldsymbol{e}_{z}, \\
\frac{\partial T}{\partial t}+(\boldsymbol{u} \cdot \nabla) T=\kappa \nabla^{2} T,
\end{gathered}
$$

where $p(x, t)$ is pressure and $\rho, v, g, \alpha$ and $\kappa$ are the mass density, kinematic viscosity, acceleration due to gravity, volumetric thermal expansivity and thermal diffusivity, respectively. Vectors $\boldsymbol{e}_{x}$ and $\boldsymbol{e}_{y}$ are mutually orthogonal unit vectors in the horizontal directions, while $\boldsymbol{e}_{z}$ is a unit vector in the vertical direction. The two no-slip and impermeable horizontal plates are positioned at $z=0$ and $z=H$, and the top (or bottom) wall surface is held at a lower (or higher) constant temperature:

$$
\boldsymbol{u}(z=0)=\boldsymbol{u}(z=H)=\mathbf{0} ; \quad T(z=0)=\Delta T>0, \quad T(z=H)=0 .
$$

The velocity and temperature fields are supposed to be periodic in the $x$ and $y$ directions with the same period $L_{x}=L_{y}=L$. The thermal convection is characterised by the Rayleigh number $R a$ and the Prandtl number Pr:

$$
R a=\frac{g \alpha \Delta T H^{3}}{\nu \kappa}, \quad \operatorname{Pr}=\frac{\nu}{\kappa} .
$$

The vertical heat flux is quantified by the Nusselt number:

$$
N u=\frac{-\kappa\langle\partial T / \partial z\rangle_{x y t}+\langle w T\rangle_{x y t}}{\kappa \Delta T / H}=1+\frac{H}{\kappa \Delta T}\langle w T\rangle_{x y z t},
$$

where $\langle\cdot\rangle_{x y t}$ and $\langle\cdot\rangle_{x y z t}$ represent the horizontal and time average and the volume and time average, respectively. The second equality is given by the volume and time average of (2.3).

Equations (2.1)-(2.3) are discretised employing a spectral Galerkin method based on the Fourier series expansion in the periodic horizontal directions and the Chebyshev polynomial expansion in the vertical direction. The nonlinear terms are evaluated using a spectral collocation method. Aliasing errors are removed with the aid of the $2 / 3$ (or 1/2) rule for the Fourier (or Chebyshev) transform. Time advancement is performed 
with the Crank-Nicholson scheme and the second-order Adams-Bashforth scheme for the diffusion terms and the rest, respectively. The nonlinear steady solutions are obtained using the Newton-Krylov iteration (for more details, see $\S 3$ and appendix A in Motoki, Kawahara \& Shimizu (2018b)).

In this paper, we present the steady solution and the turbulent states in the horizontally square periodic domain with $L / H=\pi / 2 \approx 1.57$ for $\operatorname{Pr}=1$. The domain is the same as that of the optimal states derived by Motoki et al. (2018a). The numerical process is conducted on $128^{3}$ grid points for $R a<10^{7}$ and $256^{3}$ grid points for $R a \geq 10^{7}$. In the smaller (or larger) domain with $L / H=1$ (or $L / H=2 \pi / 3.117 \approx 2.02$ ), we have confirmed that the effects of the domain size on the heat flux and the spatial structures are insignificant, which is described in the following sections. The domain size dependence is shown in appendix B with the Prandtl number dependence and the adequacy of the spatial resolution.

All the 3-D steady solutions presented in this paper satisfy the $\pi / 2$-rotation symmetry

$$
[u, v, w, T](x, y, z)=[v,-u, w, T](y,-x, z)
$$

as well as the reflection symmetry

$$
[u, v, w, T](x, y, z)=[-u, v, w, T](-x, y, z)=[u,-v, w, T](x,-y, z)
$$

and the shift-and-reflection symmetry

$$
[u, v, w, T](x, y, z)=[u, v,-w, 1-T](x+L / 2, y+L / 2,1-z) .
$$

These symmetries are not imposed on the solutions explicitly at $R a \lesssim 10^{6}$. At $R a \sim 10^{7}$, we impose the symmetries (2.7)-(2.9) in order to reduce the computational degrees of freedom.

\section{Three-dimensional steady solution}

\subsection{The $\mathrm{Nu}-\mathrm{Ra}$ scaling}

The 3-D and 2-D steady solutions in the domain with $L / H=\pi / 2$ for $\operatorname{Pr}=1$ bifurcate from the conduction state at $R a \approx 1879$, as shown in figure 1 . The $2-\mathrm{D}$ roll solution is stable up to $R a \approx 3.55 \times 10^{4}$, and subsequently a time-periodic 3 -D solution appears (the stable periodic solution has been obtained by the time evolution). Figure 2 presents $\mathrm{Nu}$ in the 3-D and 2-D steady solutions as a function of $R a$ at a higher $R a$. The red line shows the 3-D steady solution, and the open and filled circles represent the present turbulent data in the horizontally square periodic domain and the experimental data in a cylindrical container (Niemela \& Sreenivasan 2006), respectively. The blue circles represent the 2-D optimised steady solutions for $\operatorname{Pr}=1$ obtained by Sondak et al. (2015). Although the 3-D steady solution at high $R a$ exhibits smaller $N u$ than the 2-D optimised solutions, it maintains larger $N u$ than the turbulent states even at $R a \sim 10^{7}$. The inset shows $N u$ compensated by $R a^{\gamma}$. The scaling exponent $\gamma$ of turbulent states shows $2 / 7$ for $R a \lesssim 10^{7}$; at higher $R a$, it changes to 0.31 . Such a transition has been experimentally and numerically observed for $\mathrm{Pr} \sim 1$ (Castaing et al. 1989; Silano, Sreenivasan \& Verzicco 2010). The exponent of the heat flux in the 3 -D steady solution is greater than $2 / 7$ but less than $1 / 3$, with 0.31 being the closest, whereas that in the 2 -D steady solution with fixed $L / H=\pi / 2$ (blue line) is smaller than $2 / 7$. The bumps in the 3 -D steady solution correspond to the remarkable changes in the thermal and flow structures, which will be shown in $\S 3.3$. At $R a \sim 10^{4}$ the heat flux of the 3-D steady solution surpasses maxima in 


\section{S. Motoki, G. Kawahara and M. Shimizu}

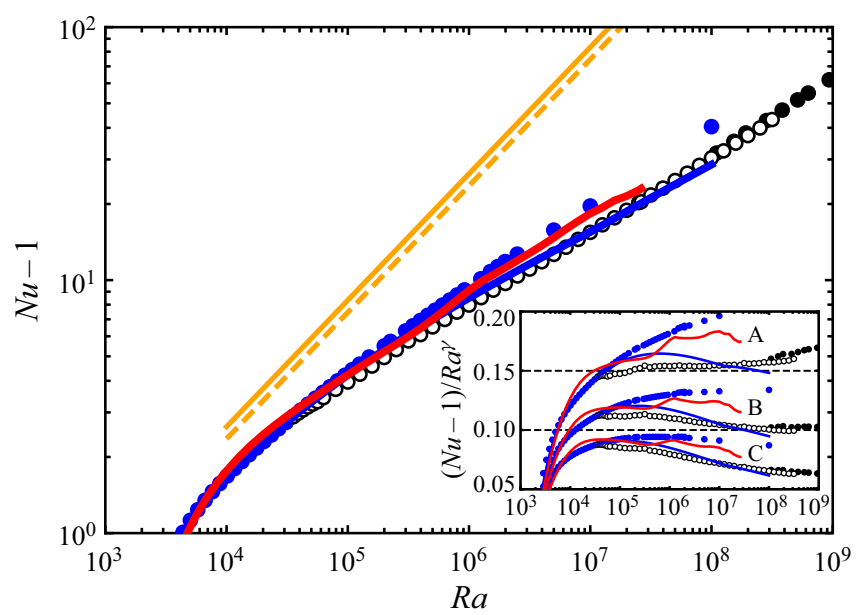

Figure 2. Nusselt number $\mathrm{Nu}-1$ as a function of Rayleigh number $\mathrm{Ra}$. The red and blue solid lines represent the 3-D and 2-D steady solutions, respectively. The open black circles represent the present turbulent data obtained in the horizontally square periodic domain, and the filled ones are the experimental turbulent data in a cylindrical container (Niemela \& Sreenivasan 2006). The blue filled circles indicate 2-D optimised steady solutions for $\operatorname{Pr}=1$ obtained by Sondak et al. (2015). The orange solid and dashed lines indicate the upper bound $N u-1=0.02634 R a^{1 / 2}$ (Plasting \& Kerswell 2003) and the optimal scaling $N u-1=0.0236 R a^{1 / 2}$, respectively, evaluated from the wall-to-wall optimal transport states (Motoki et al. 2018a). The inset shows $\mathrm{Nu}$ compensated by $R a^{\gamma}: \gamma=2 / 7$ (plot A); $\gamma=0.31$ (plot B); $\gamma=1 / 3$ (plot C).

the 2-D steady solutions. As shown in appendix B, the 3-D steady solution with the smaller horizontal period $(L / H=1)$ shows larger $N u$ at $R a \sim 10^{5}$. The results imply that a family of the 3-D steady solutions with optimised horizontal periods might achieve maximal heat transfer in the steady Rayleigh-Bénard convection. The orange dashed line indicates the optimal scaling $N u-1=0.0236 R a^{1 / 2}$ (Motoki et al. 2018a) given by the 3-D optimal states maximising the wall-to-wall transport, and it is quite close to the rigorous upper bound, $N u-1=0.02634 R a^{1 / 2}$ (Plasting \& Kerswell 2003). Although the 3-D optimal states exhibiting significantly high heat flux require an external body force different from buoyancy, the optimal state can be continuously connected to the present 3-D steady solution of the full Boussinesq equations by a homotopy from the body force to the buoyancy, as shown in appendix A.

\subsection{Mean temperature and root-mean-square profiles}

Figure 3 compares the mean temperature $\langle T\rangle_{x y t}$, root-mean-square (RMS) temperature $T_{r m s}=\left\langle\left(T-\langle T\rangle_{x y t}\right)^{2}\right\rangle_{x y t}^{1 / 2}$ and RMS vertical velocity $w_{r m s}=\left\langle w^{2}\right\rangle_{x y t}^{1 / 2}$ for the 3-D steady solution with those for the turbulent states at $10^{5} \leq R a \leq 10^{7}$. In figure $3(b, d, f)$ the distance to the wall, $z$, is normalised by $\delta$, where $\delta$ is the thermal conduction layer thickness given by $\delta / H=1 /(2 \mathrm{Nu})$. The mean temperature in the 3 -D steady solution well follows that in the turbulent states; furthermore, the corresponding RMS values are also in good agreement with each other. In the bulk region, all mean temperature profiles are flattened, as a result of the nearly complete mixing by large-scale circulation. The temperature difference $\Delta T / 2$ exists only at the thermal conduction layer, $0 \leq z \lesssim 2 \delta / H=1 / N u$, and $T_{r m s}$ exhibits a peak at $z / \delta \approx 1$. If the advection, diffusion and buoyancy terms in the 
Multi-scale steady solution for Rayleigh-Bénard convection
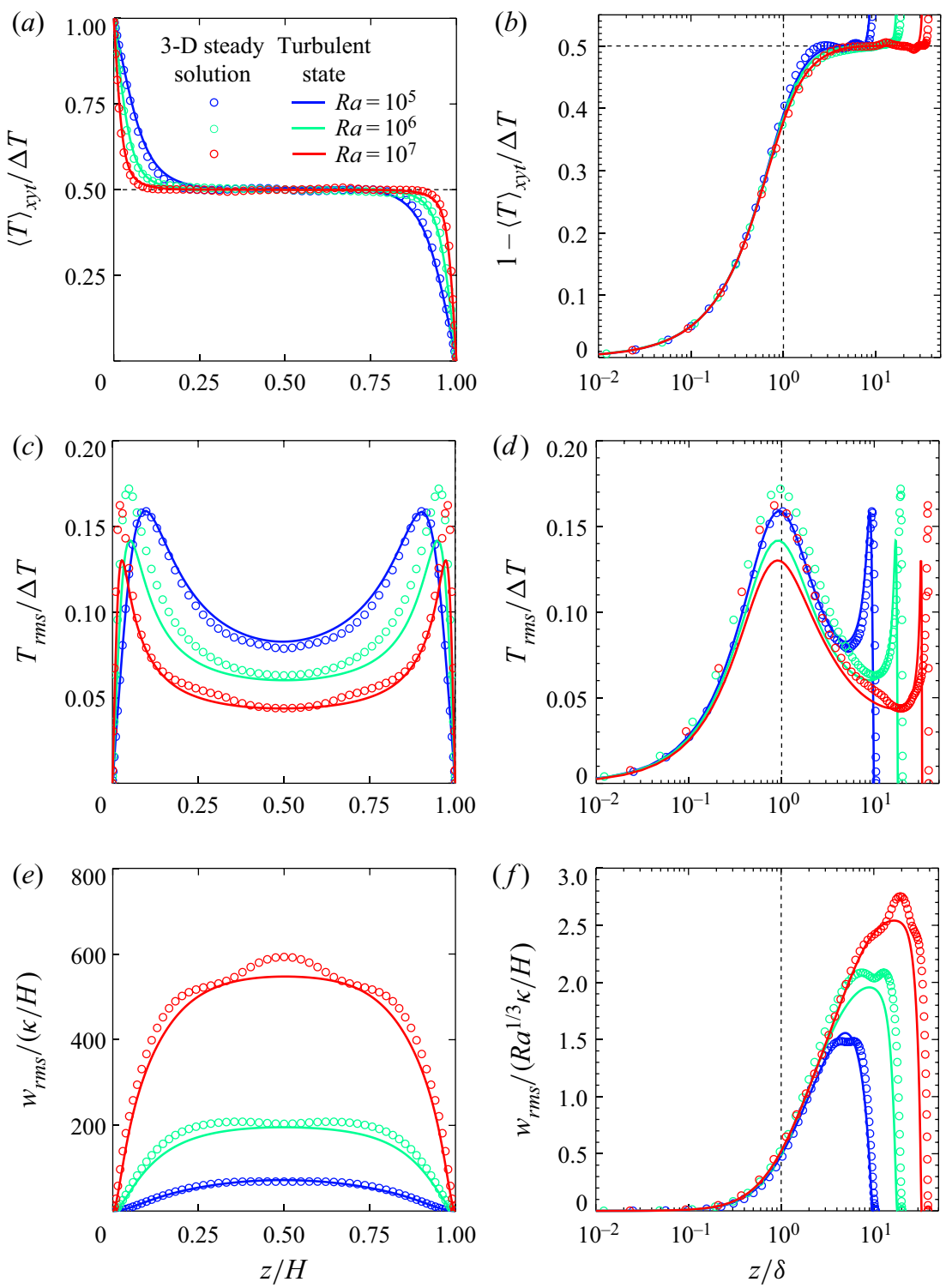

Figure 3. ( $a, b)$ Mean temperature, $(c, d)$ RMS temperature and $(e, f)$ RMS vertical velocity as a function of $(a, c, e) z / H$ and $(b, d, f) z / \delta$ in the 3-D steady solution and the turbulent state. Here $\delta$ is the thermal conduction layer thickness that scales as $\delta / H=1 /(2 N u)$.

Navier-Stokes equation (2.2) at the conduction layer are balanced as

$$
\frac{w^{\prime 2}}{\delta} \sim v \frac{w^{\prime}}{\delta^{2}} \sim g \alpha \Delta T
$$

(the balance between the advection and diffusion terms is given by that in the energy equation (2.3) for $v \sim \kappa)$, the near-wall vertical velocity would then be

$$
w^{\prime} \sim(g \alpha \Delta T \delta)^{1 / 2} \sim R a^{1 / 3} \frac{\kappa}{H}
$$




\section{S. Motoki, G. Kawahara and M. Shimizu}

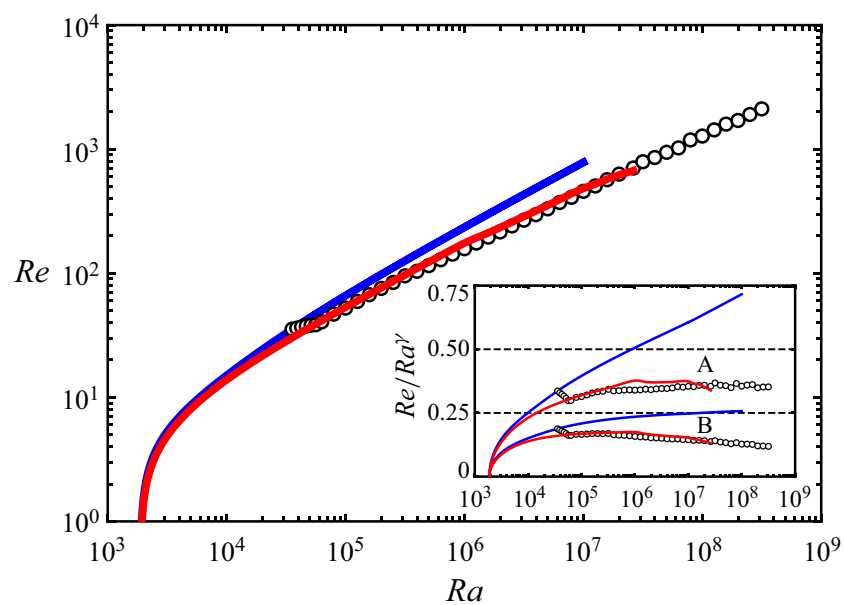

Figure 4. Reynolds number $R e$ as a function of Rayleigh number $R a$. The red and blue solid lines represent the 3-D and 2-D steady solutions, respectively. The open circles represent the present turbulent data obtained in the horizontally square periodic domain. The inset shows $N u$ compensated by $R a^{\gamma}: \gamma=4 / 9$ (plot A); $\gamma=1 / 2$ (plot B).

yielding the scaling law

$$
\mathrm{Nu} \sim R a^{1 / 3}
$$

(Kawano et al. 2021), which has been given by Malkus' theory (Malkus 1954) and the argument of Grossmann \& Lohse (2000). As shown in figure 3( $f$ ), the RMS vertical velocity $w_{r m s}$ scales as $R a^{1 / 3} \kappa / H$ near the wall $z / \delta \sim 1$.

In the bulk region, where the effects of viscosity or thermal conduction are insignificant, the advection and buoyancy terms are balanced as

$$
\frac{U_{b}^{2}}{H} \sim g \alpha \Delta T^{\prime},
$$

and the dominance of convective heat transfer in bulk and the scaling (3.3) give us

$$
\frac{U_{b} \Delta T^{\prime}}{\kappa \Delta T / H} \sim R a^{1 / 3}
$$

(Kawano et al. 2021). Equations (3.4) and (3.5) yield the bulk velocity scales as

$$
U_{b} \sim \operatorname{Pr}^{-2 / 3} \operatorname{Ra}^{4 / 9} v / H .
$$

The Reynolds number based on $U_{b}$ scales with $\mathrm{Pr}^{-2 / 3} \mathrm{Ra}^{4 / 9}$, exhibiting consistency with the scaling suggested by Grossmann \& Lohse (2000) when both energy and scalar dissipation are bulk-dominated. Figure 4 shows the Reynolds number Re defined as

$$
\operatorname{Re}=\frac{\left\langle w^{2}\right\rangle_{x y z t}^{1 / 2} H}{v}
$$

as a function of $R a$. The Reynolds number $R e$ of the 3-D steady solution is comparable with that of the turbulent states exhibiting $R e \sim R a^{4 / 9}$ at a high $R a$. In contrast, the 2-D steady solution with fixed $L / H=\pi / 2$ seems to approach $R e \sim R a^{1 / 2}$, implying that $\left\langle w^{2}\right\rangle_{x y z t}^{1 / 2}$ scales with the buoyancy-induced terminal velocity $U=(g \alpha \Delta T H)^{1 / 2}$. 

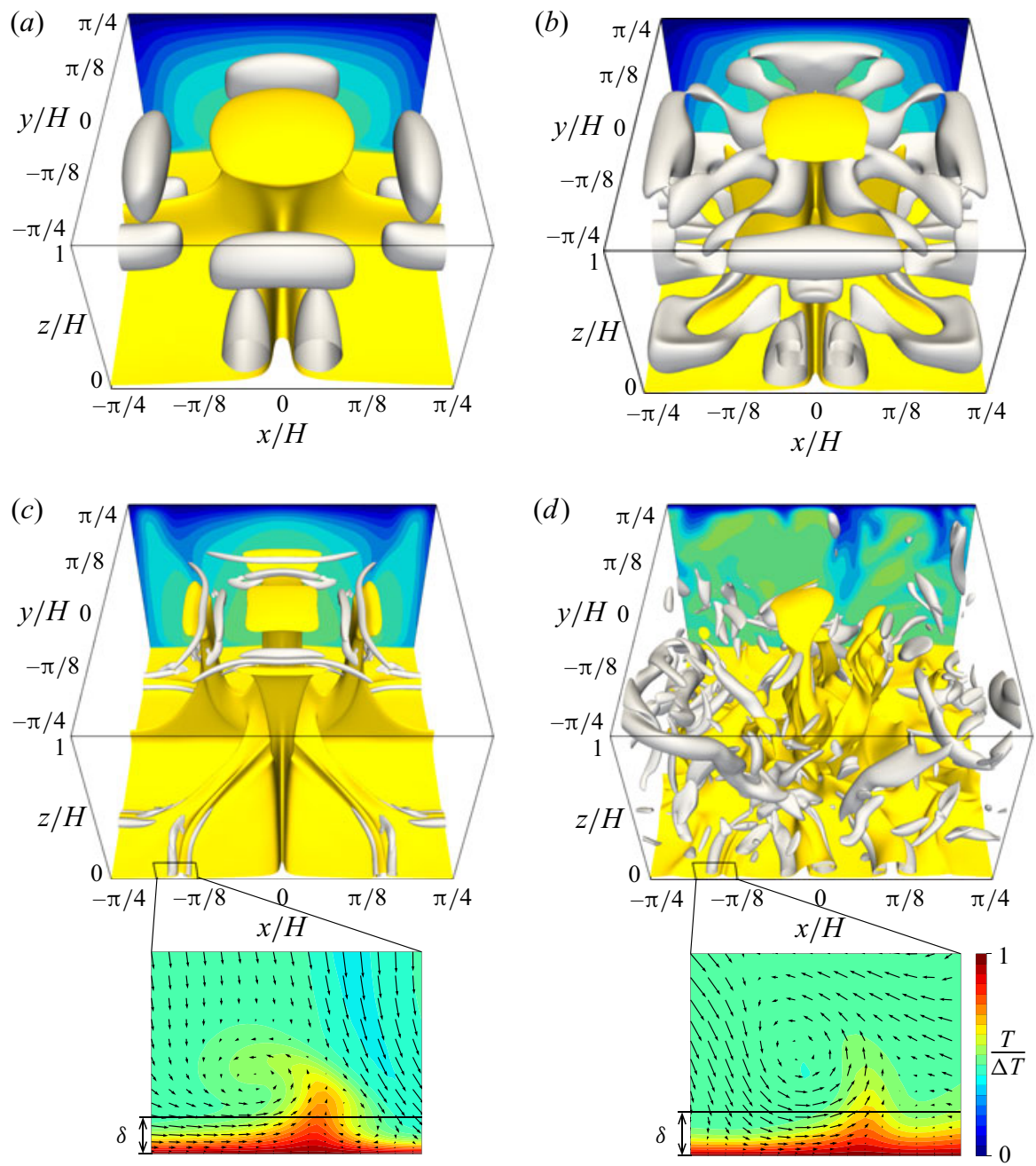

Figure 5. Thermal and flow structures in $(a-c)$ the 3-D steady solution and $(d)$ the turbulent state at $(a) R a=$ $10^{5}$, (b) $R a=10^{6}$ and $(c, d) R a=10^{7}$. The yellow and grey objects represent the isosurfaces of the temperature $T / \Delta T=0.6$ and the positive second invariant of the velocity gradient tensor, $(a) Q /\left(\kappa^{2} / H^{4}\right)=1.28 \times 10^{5}$, (b) $Q /\left(\kappa^{2} / H^{4}\right)=1.28 \times 10^{6}$ and $(c, d) \quad Q /\left(\kappa^{2} / H^{4}\right)=8 \times 10^{7}$, respectively. The contours represent temperature $T$ in the plane $y / H=\pi / 4(=-\pi / 4)$, and the velocity vectors $(u, w)$ in the enlarged views in $(c, d)$ are superposed.

\subsection{Thermal and flow structures}

Figure 5 visualises the thermal and flow structures in the 3-D steady solution and the turbulent state. The yellow objects represent the isosurfaces of temperature $T / \Delta T=0.6$, representing high-temperature plumes; the grey objects represent the vortex structures visualised by the positive second invariant of the velocity gradient tensor

$$
Q=-\frac{1}{2} \frac{\partial u_{i}}{\partial x_{j}} \frac{\partial u_{j}}{\partial x_{i}}
$$

At $R a=10^{5}$ (figure $5 a$ ), the 3-D steady solution consists of large-scale thermal plumes as well as accompanying near-wall small-scale plume and vortex structures near the 


\section{S. Motoki, G. Kawahara and M. Shimizu}

vertical planes $x / H=0$ and $y / H=0$. As $R a$ for the 3 -D steady solution increases, smaller thermal plume structures (and relevant smaller and stronger tube-like vortex structures) appear near the walls without affecting the already existing large-scale structures. As $\mathrm{Ra}$ increases from $10^{5}$ to $10^{6}$, thermal and vortex structures newly emerge along the diagonals $y= \pm x$ near the walls (figure $5 b$ ). As $R a$ increases further from $10^{6}$ to $10^{7}$, smaller-scale structures appear along $x / H= \pm \pi / 8$ and $y / H= \pm \pi / 8$ near the walls, and the large secondary plumes approach the opposite wall (figure $5 \mathrm{c}$ ). The bumps of $\mathrm{Nu}$ seen in figure 2 correspond to these remarkable changes in the thermal and vortex structures.

At $R a=10^{7}$ (figure $5 c$ ), we observe sheet-like thermal plumes with the smallest-scale vortices, which are quite similar to those observed in the snapshot of the turbulent state (figure $5 d$ ). The smallest-scale structures appear in the thermal conduction layer, and the sizes of the plumes and vortices scale with the thickness $\delta$. In 2-D optimised steady solutions (Sondak et al. 2015; Waleffe et al. 2015), the appearance of small-scale thermal and vortex structures has been observed with the shrinking horizontal period as $R a$ increases, and the scaling $N u \sim R a^{0.31}$ is achieved by a family of solutions with smaller horizontal periods. It should be stressed that the single 3-D steady solution spontaneously reproduces the multi-scale coherent structures of convective turbulence.

\section{Hierarchical vortices and energy transfer in wavenumber space}

The 3-D steady solution is found to reproduce the near-wall coherent structures in convective turbulence; however, the flow in bulk should be addressed. The developed turbulence organises hierarchical coherent vortex structures of various scales (Goto, Saito \& Kawahara 2017; Motoori \& Goto 2019). The smallest-scale vortex structures can still be extracted by employing the isosurface of $Q$, as shown in figure 5; however, it is difficult to identify large- and intermediate-scale structures. To examine the hierarchy of multi-scale vortices in the 3-D steady solution, we consider coarse-graining the velocity field $\boldsymbol{u}$. The coarse-grained velocity field $\boldsymbol{u}^{*}$ is obtained using the Gaussian low-pass filter (Lozano-Durán, Holzner \& Jimenez 2016; Motoori \& Goto 2019) as follows:

$$
\boldsymbol{u}^{*}(\boldsymbol{x})=\int_{V} a \boldsymbol{u}\left(\boldsymbol{x}^{\prime}\right) \exp \left\{-\left(\frac{\pi \Delta r}{\sigma}\right)^{2}\right\} \mathrm{d} \boldsymbol{x}^{\prime}
$$

where $\Delta r=\left|x^{\prime}-\boldsymbol{x}\right|, \sigma$ is the filter width and $a$ is a constant such that the integral of the kernel over the control volume $V$ is unity. In the wall-normal direction, the Gaussian filter is applied by reflecting it at the wall (Lozano-Durán et al. 2016). Figure 6 shows hierarchical vortex structures in the $3-\mathrm{D}$ steady solution at $R a=2.6 \times 10^{7}$. Non-filtered structures are shown in figure 6(a), and the isosurfaces of $Q$ of the filtered velocity $\boldsymbol{u}^{*}$ with $\sigma=H(=2 L / \pi), L / 2, L / 4, L / 8$ and $L / 16$ are displayed in figure $6(b-f)$, respectively. The blue objects in figure $6(b)$ are the largest-scale structures corresponding to the large-scale circulation, whereas the red ones in figure $6(f)$ are the smallest-scale structures of size $\sigma / 2=L / 32 \approx 2 \delta$, which coincides with the size of vortices observed in the non-filtered field (figure $6 a$ ). The light blue, green and light red objects in figure $6(c, d, e)$ illustrate the intermediate-scale vortex structures of eight, four and two times the size of the smallest-scale vortices, respectively. The smaller-scale vortex structures exist closer to the wall, while the intermediate-scale ones are observed in the bulk region in figure $6(d, e)$.

Figure $6(g, h)$ shows the superposed structures, and from their spatial distribution, it is conjectured that the bulk flow is composed of multi-scale coherent structures. Figure $7(a)$ shows the energy spectrum $E(k, z)$ of the 3-D steady solution at the centre of the fluid 
Multi-scale steady solution for Rayleigh-Bénard convection
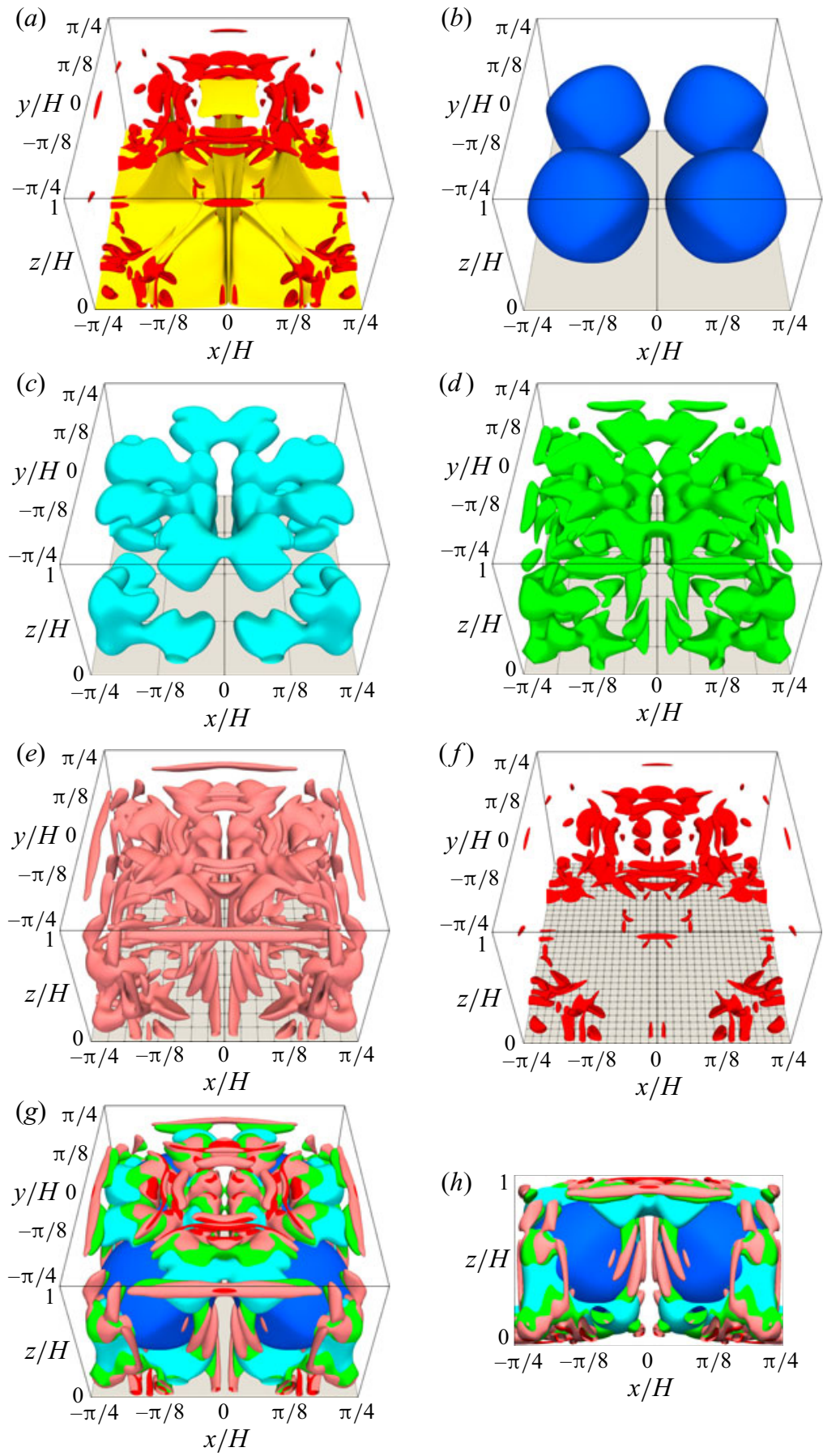

Figure 6. Hierarchical vortex structures visualised by coarse-graining with Gaussian low-pass filter. (a) The yellow and red objects represent the isosurfaces of the non-filtered $T / \Delta T=0.6$ and $Q /\left(\kappa^{2} / H^{4}\right)=2 \times 10^{8}$, respectively. $(b-h)$ The vortex structures are visualised by the isosurfaces of $Q /\left(\kappa^{2} / H^{4}\right)$ of the filtered velocity field with filter widths of $\sigma=H(=2 L / \pi$ ) (blue), $\sigma=L / 4$ (light blue), $\sigma=L / 8$ (green), $\sigma=L / 16$ (light red) and $\sigma=L / 32$ (red); they are superposed in $(g, h)$. The isosurface levels are (blue) $5 \times 10^{5}$, (light blue) $4 \times 10^{6}$, (green) $1.2 \times 10^{7}$, (light red) $3 \times 10^{7}$ and (red) $1.6 \times 10^{8}$. 


\section{S. Motoki, G. Kawahara and M. Shimizu}
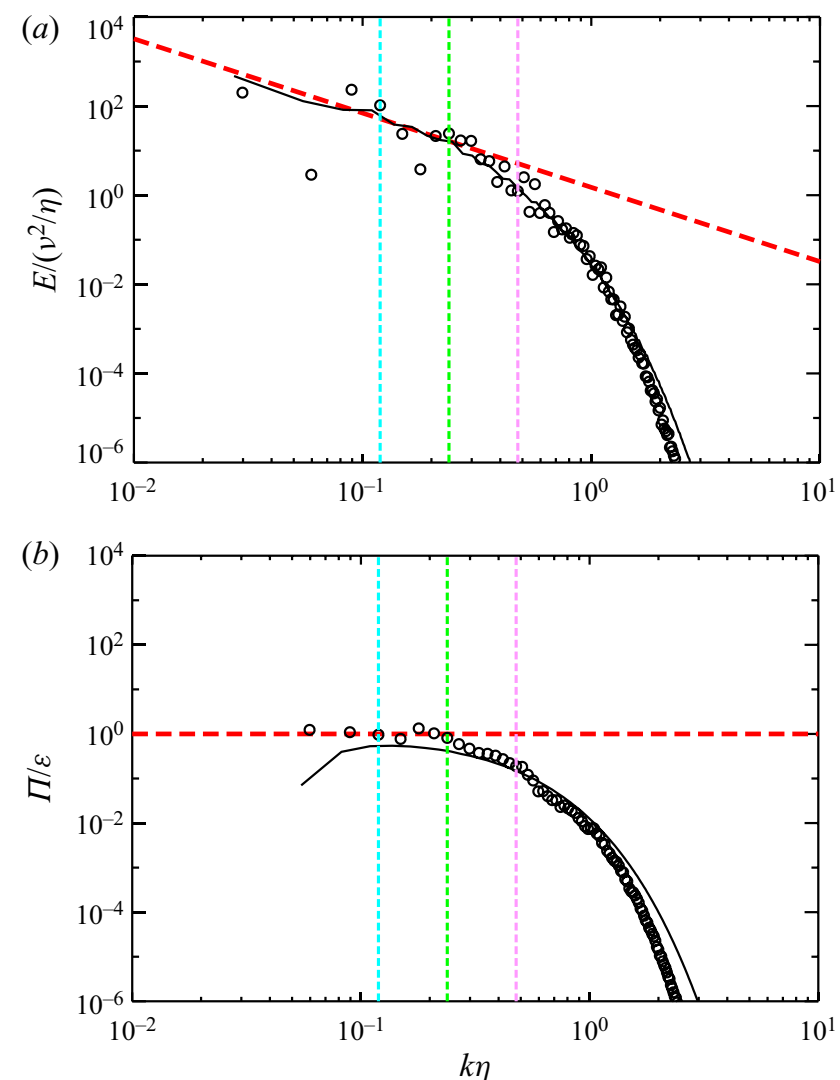

Figure 7. (a) Energy spectrum $E$ and (b) energy flux $\Pi$ at the centre of the fluid layer, $z=H / 2$, in the 3-D steady solution (circles) and the turbulent state (lines) at $R a=2.6 \times 10^{7}$. The lateral and longitudinal axes are normalised by the kinematic viscosity $v$ and the energy dissipation rate $\varepsilon$ at $z=H / 2$, which yield the Kolmogorov micro-scale length $\eta=\left(v^{3} / \varepsilon\right)^{1 / 4}$. The red dashed lines represent $E=1.5 \varepsilon^{2 / 3} k^{-5 / 3}$ and $\Pi / \varepsilon=1$, respectively. The light blue, green and light red colours indicate $k=2 \pi /(L / 4), 2 \pi /(L / 8)$ and $2 \pi /(L / 16)$, respectively, normalised with $\eta$ in the 3-D steady solution, corresponding to the intermediate-scale structures shown in figure 6.

layer, $z=H / 2$, and the corresponding turbulence spectrum at $R a=2.6 \times 10^{7}$. The energy spectrum $E(k, z)$ is defined as

$$
E(k, z)=\frac{L}{2 \pi} \sum_{k-\Delta k / 2<\left|\boldsymbol{k}_{2 D}\right|<k+\Delta k / 2} \frac{1}{2}\left\langle\left|\tilde{\boldsymbol{u}}\left(\boldsymbol{k}_{2 D}, z\right)\right|^{2}\right\rangle_{t},
$$

where $(\tilde{\cdot})$ indicates the Fourier coefficients in the periodic $(x$ and $y)$ directions and $\langle\cdot\rangle_{t}$ represents the time average. Here $\boldsymbol{k}_{2 D}=\left(k_{x}, k_{y}\right)$ and $k=\left|\boldsymbol{k}_{2 D}\right|$ are the wavenumber vector and its magnitude, respectively, and $\Delta k=2 \pi / L$. The lateral and longitudinal axes are normalised by the kinematic viscosity $v$ and the energy dissipation rate

$$
\varepsilon(z)=\frac{v}{2}\left\langle\left(\frac{\partial u_{i}}{\partial x_{j}}+\frac{\partial u_{j}}{\partial x_{i}}\right)^{2}\right\rangle_{x y t},
$$

which yield the Kolmogorov micro-scale length $\eta=\left(v^{3} / \varepsilon\right)^{1 / 4}$. The spectra of the 3-D steady solution and turbulent state are in good agreement at high wavenumber $k \eta \gtrsim 10^{0}$. 
Furthermore, in the wavenumber band $2 \pi /(L / 4) \lesssim k \eta \lesssim 2 \pi /(L / 16)$, corresponding to the intermediate-scale range, the energy spectrum follows Kolmogorov's $-5 / 3$ power law, $E=C_{K} \varepsilon^{2 / 3} k^{-5 / 3}$ (Kolmogorov 1941), with the constant $C_{K} \approx 1.5$, which is consistent with that in the inertial subrange of high-Reynolds-number turbulence (Sreenivasan 1995; Ishihara et al. 2016).

In figure $7(b)$, we show the energy flux in the wavenumber space, $\Pi(k, z)$ (Mizuno 2016), defined as

$$
\begin{gathered}
\Pi(k, z)=\sum_{k^{\prime} \geq k-\Delta k / 2<\left|\boldsymbol{k}_{2 D}\right|<k+\Delta k / 2} T^{S}\left(\boldsymbol{k}_{2 D}, z\right), \\
T^{s}\left(\boldsymbol{k}_{2 D}, z\right)=\operatorname{Re}\left[\left\langle\partial_{j} \tilde{u}_{i}\left(\widetilde{u_{i} u_{j}}\right)^{\dagger}\right\rangle_{t}-\frac{1}{2} \frac{\left.\partial\left\langle\tilde{u}_{j}\left(\widetilde{u_{j} w}\right)^{\dagger}\right\rangle_{t}\right]}{\partial z}\right],
\end{gathered}
$$

where $\left(\partial_{1}, \partial_{2}, \partial_{3}\right)=\left(i k_{x}, \mathrm{i} k_{y}, \partial / \partial z\right)$ and $\dagger$ denotes the complex conjugate. Here $T^{s}\left(\boldsymbol{k}_{2 D}, z\right)$ represents the energy transfer between the Fourier modes, and the sum of all spectral components does not contribute to the total energy budget, i.e. $\sum_{k_{2 D}} T^{S}\left(\boldsymbol{k}_{2 D}, z\right)=0$. In the intermediate-scale range, the energy flux exhibits positive values, that is, the energy is transferred from large to small scale, and it scales with the same order of energy dissipation rate.

In the optimised 2-D steady solution (Sondak et al. 2015; Waleffe et al. 2015), as $R a$ increases the horizontal scale of large-scale convective rolls reduces due to the shrinking of the horizontal period. The bulk flows at high $R a$ diverge from those in the turbulent states, although it is intriguing that the optimised steady solutions exhibit the turbulent scaling $N u \sim R a^{0.31}$. Meanwhile, the 3-D steady solution reproduces not only the near-wall coherent structures but also the turbulence in the bulk of the Rayleigh-Bénard convection.

\section{Summary and conclusions}

We have discovered a 3-D steady solution to the Boussinesq equations, exhibiting a scaling of $N u \sim R a^{0.31}$ and leading to multi-scale coherent structures, which are similar to those observed in turbulent Rayleigh-Bénard convection. The invariant solution bifurcates from the conduction state at $R a \sim 10^{3}$, and it has been tracked up to $R a \sim 10^{7}$ using the Newton-Krylov iteration. The heat flux of the 3-D steady solution can surpass maxima in the 2-D steady solutions (Sondak et al. 2015; Waleffe et al. 2015), and thus a family of the 3-D steady solutions with optimised horizontal periods might achieve maximal heat transfer in steady Rayleigh-Bénard convection. In contrast, in the 3-D steady solution with fixed horizontal periods, the horizontal-averaged temperature and the RMS of the temperature and velocity fluctuations are in good agreement with the horizontal and temporal averages obtained for the turbulent states. In the near-wall region of both the 3-D steady solution and the turbulent states, smaller-scale thermal plumes emerge with an increase in $R a$. The size of the coherent thermal structures and relevant vortices is comparable with the thermal conduction layer thickness $\delta / H=1 /(2 N u)$, and the RMS vertical velocity at $z / \delta \sim 1$ scales with the velocity scale $R a^{1 / 3} \kappa / H$, corresponding to $N u \sim R a^{1 / 3}$. The Reynolds number $R e$ based on the RMS vertical velocity scales as $R e \sim R a^{4 / 9}$, which is related to the scaling $N u \sim R a^{1 / 3}$. The bulk flow in the 3-D steady solution comprises hierarchical multi-scale vortices. We have extracted the large- and intermediate-scale vortex structures by employing the coarse-graining method. The ratio of the largest to the smallest length scales in the 3 -D steady solution at $R a=2.6 \times 10^{7}$ 


\section{S. Motoki, G. Kawahara and M. Shimizu}

is approximately 20 . The energy spectrum at the centre of the fluid layer shows good agreement with that of the turbulent state. In the intermediate-scale range, the spectrum follows $E=1.5 \varepsilon^{2 / 3} k^{-5 / 3}$, which is commonly observed in the inertial subrange of the developed turbulence. Furthermore, energy is transferred from large to small scales in the wavenumber space, and the energy flux balances the energy dissipation rate, in accordance with the Kolmogorov-Obukhov energy cascade view.

Recently, van Veen, Vela-Martín \& Kawahara (2019) have found a time-periodic solution that reproduces inertial range dynamics in a triply periodic turbulence driven by a constant body force of the Taylor-Green type. They have obtained the invariant solution by applying large-eddy simulation based on the Smagorinsky-type eddy-viscosity model. Meanwhile, by introducing the buoyant force, we have succeeded in finding a multi-scale solution of the full incompressible Navier-Stokes equations without any empirical models. We believe that the current work and approaches based on multi-scale invariant solutions will trigger significant advances in the theoretical understanding and deductive modelling of coherent structures and energy transfer mechanisms in developed turbulence.

Acknowledgements. We are grateful to Dr D. Sondak (Harvard University) for providing us with numerical data.

Funding. This work was supported by the Japanese Society for Promotion of Science (JSPS) KAKENHI (grant nos. 19K14889 and 18H01370). In this research work we used the supercomputer of ACCMS, Kyoto University. This work was supported by NIFS Collaboration Research Program (NIFS19KNSS124).

Declaration of interests. The authors report no conflict of interest.

Author ORCIDs.

(D) Shingo Motoki https://orcid.org/0000-0002-2268-412X.

\section{Appendix A. Homotopy from wall-to-wall optimal transport solution}

The wall-to-wall optimal transport problem (Hassanzadeh et al. 2014; Motoki et al. 2018a; Souza et al. 2020) involves maximising the heat flux between two parallel plates with a constant temperature difference, under the constraint of fixed total enstrophy, which is written as

$$
\left.\begin{array}{ll}
\text { Maximise } & N u=1+\langle w \theta\rangle_{x y z}, \\
\text { subject to } & \boldsymbol{\nabla} \cdot \boldsymbol{u}=0, \\
& (\boldsymbol{u} \cdot \boldsymbol{\nabla}) \theta+w=\kappa \nabla^{2} \theta, \\
& P e=\frac{\left\langle|\boldsymbol{\nabla} \cdot \boldsymbol{u}|^{2}\right\rangle_{x y z}^{1 / 2} H^{2}}{\kappa}=\text { const. } \\
& \text { and the boundary conditions, }
\end{array}\right\}
$$

where $\theta=T-(1-z)$ is the temperature fluctuation about a conduction state. The constraint optimisation is relevant to the maximisation of the objective functional

$$
\mathcal{F}^{\prime}=\left\langle w^{\prime} \theta^{\prime}-\phi^{\prime}\left(\boldsymbol{x}^{\prime}\right)\left[\left(\boldsymbol{u}^{\prime} \cdot \nabla^{\prime}\right) \theta^{\prime}+w^{\prime}-\nabla^{\prime 2} \theta^{\prime}\right]+\psi^{\prime}\left(\boldsymbol{x}^{\prime}\right)\left(\nabla^{\prime} \cdot \boldsymbol{u}^{\prime}\right)+\frac{\mu^{\prime}}{2}\left(P e^{2}-\left|\nabla^{\prime} \boldsymbol{u}^{\prime}\right|^{2}\right)\right\rangle_{x y z}
$$




\section{Multi-scale steady solution for Rayleigh-Bénard convection}
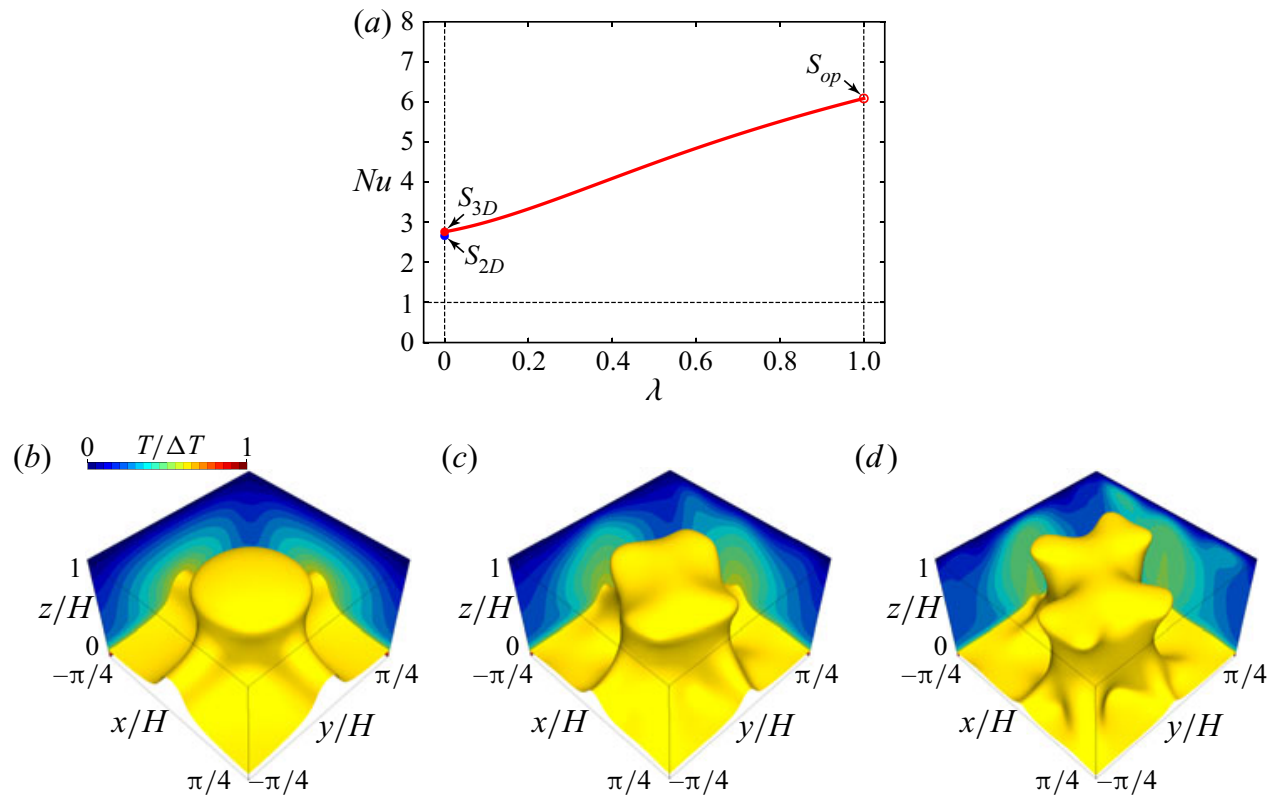

Figure 8. Homotopy from the wall-to-wall optimal transport solution at $P e=508$ (from Motoki et al. 2018a) to the present 3-D steady solution for a fixed $R a=10^{4}$ and $\operatorname{Pr}=1$. (a) Nusselt number $N u$ as a function of the homotopy parameter $\lambda$. The red open circle shows the optimal solution $S_{o p}$ of the Euler-Lagrange equations for the wall-to-wall optimal transport problem, and the red and blue filled circles represent the 3-D steady solution $S_{3 D}$ and the 2-D steady solution $S_{2 D}$ of the Boussinesq equations, respectively. $(b-d)$ Isosurfaces of temperature $T / \Delta T=0.6$ at $(b) \lambda=0,(c) \lambda=0.3$ and $(d) \lambda=1.0$. The contours represent the temperature $T$ in the planes $x / H=-\pi / 4$ and $y / H=\pi / 4$. The numerical computation is carried out on $64^{3}$ grid points.

where $\phi^{\prime}\left(\boldsymbol{x}^{\prime}\right), \psi^{\prime}\left(\boldsymbol{x}^{\prime}\right)$ and $\mu^{\prime}$ are Lagrange multipliers and prime $(\cdot)^{\prime}$ represents a non-dimensional variable based on $H, \Delta T, \kappa$ and $\rho$. The Euler-Lagrange equations are

$$
\begin{gathered}
\frac{\delta \mathcal{F}^{\prime}}{\delta \boldsymbol{u}^{\prime}} \equiv-\nabla^{\prime} \psi^{\prime}+\theta^{\prime} \nabla^{\prime} \phi^{\prime}+\mu^{\prime} \nabla^{\prime 2} \boldsymbol{u}^{\prime}+\left(\theta^{\prime}+\phi^{\prime}\right) \boldsymbol{e}_{z}=\mathbf{0}, \\
\frac{\delta \mathcal{F}^{\prime}}{\delta \theta^{\prime}} \equiv\left(\boldsymbol{u}^{\prime} \cdot \nabla^{\prime}\right) \phi^{\prime}+w^{\prime}+\nabla^{\prime 2} \phi^{\prime}=0, \\
\frac{\delta \mathcal{F}^{\prime}}{\delta \phi^{\prime}} \equiv-\left(\boldsymbol{u}^{\prime} \cdot \nabla^{\prime}\right) \theta^{\prime}+w^{\prime}+\nabla^{\prime 2} \theta^{\prime}=0, \\
\frac{\delta \mathcal{F}^{\prime}}{\delta \psi^{\prime}} \equiv \nabla^{\prime} \cdot \boldsymbol{u}^{\prime}=0, \\
\frac{\partial \mathcal{F}^{\prime}}{\partial \mu^{\prime}} \equiv \frac{1}{2}\left\langle P e^{2}-\left|\nabla^{\prime} \boldsymbol{u}^{\prime}\right|^{2}\right\rangle_{x y z}=0 .
\end{gathered}
$$

In our previous work (Motoki et al. 2018a), we obtained the optimal state to satisfy (A3)-(A7). Thus, to fulfil the Boussinesq equations, the optimal velocity and temperature field $\left(\boldsymbol{u}_{o p}^{\prime}, \theta_{o p}^{\prime}\right)$ require an additional body force,

$$
\boldsymbol{f}^{\prime}\left(\boldsymbol{x}^{\prime}\right)=-\left(\boldsymbol{u}_{o p}^{\prime} \cdot \nabla^{\prime}\right) \boldsymbol{u}_{o p}^{\prime}-\nabla^{\prime} p_{o p}^{\prime}+\operatorname{Pr} \nabla^{\prime 2} \boldsymbol{u}_{o p}^{\prime}+\operatorname{PrRa}\left(1-z^{\prime}+\theta_{o p}^{\prime}\right) \boldsymbol{e}_{z},
$$




\section{S. Motoki, G. Kawahara and M. Shimizu}
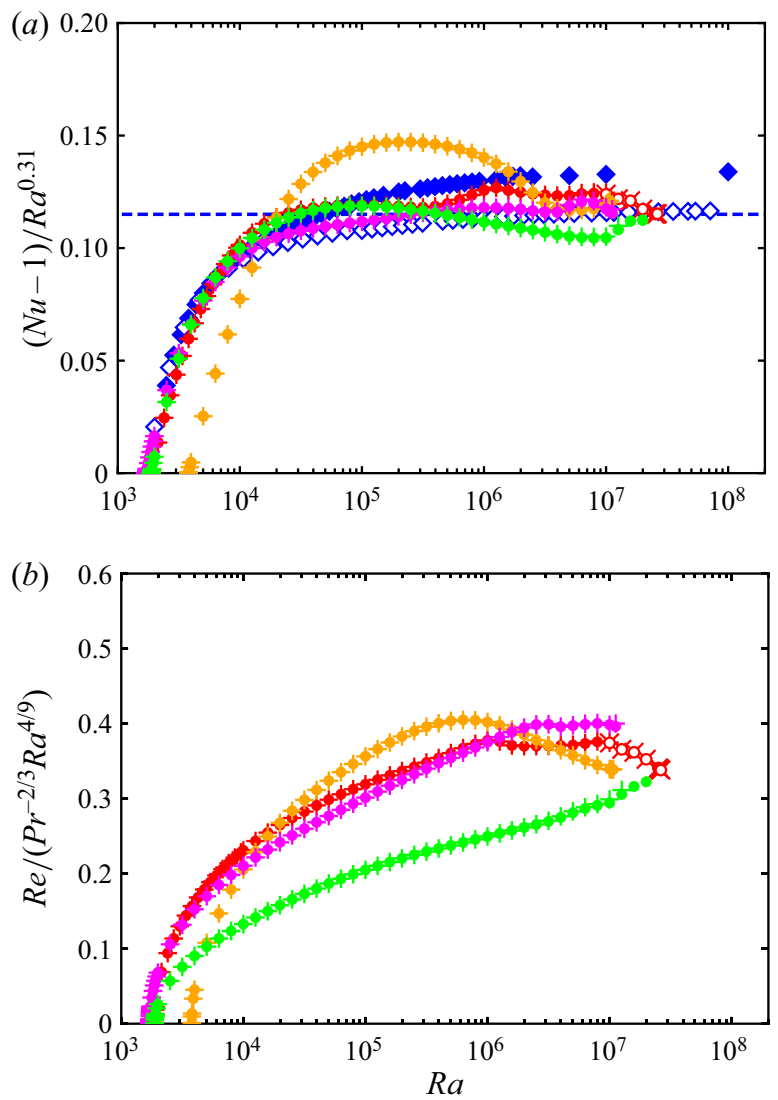

Figure 9. (a) Nusselt number $N u$ compensated by $R a^{0.31}$ and (b) Reynolds number $R e$ compensated by $\mathrm{Pr}^{-2 / 3} \mathrm{Ra}^{4 / 9}$ as a function of the Rayleigh number $\mathrm{Ra}$ in the 3-D steady solutions for different horizontal period $L$ and Prandtl number $P r$. The magenta, red and orange symbols represent $L / H=2 \pi / 3.117, \pi / 2$ and 1, respectively, for $\operatorname{Pr}=1$, and the green symbols represent $L / H=\pi / 2$ for $\operatorname{Pr}=7$. The blue filled and open diamonds represent the 2-D optimised steady solutions (Sondak et al. 2015) for $\operatorname{Pr}=1$ and 7, respectively. The blue dashed line indicates the least square fit in the 2-D steady solutions for $\operatorname{Pr}=7, \mathrm{Nu}-1=0.115 \mathrm{Ra} a^{0.31}$, determined in the range $10^{7}<R a \leq 10^{9}$ (Waleffe et al. 2015). The present solutions have been obtained on grid points of,$+\left(N_{x}, N_{y}, N_{z}\right)=(64,64,64) ; \bullet,(128,128,128) ; \times,(192,192,128) ;$,,$(256,256,256)$.

which is different from the buoyant force, where $p_{o p}^{\prime}$ is the pressure determined by the Poisson equation stemming from the Boussinesq equations. We consider homotopy from the Euler-Lagrange system to the steady Boussinesq system:

$$
\begin{gathered}
-\left(\boldsymbol{u}^{\prime} \cdot \nabla^{\prime}\right) \boldsymbol{u}^{\prime}-\nabla^{\prime} p^{\prime}+\operatorname{Pr} \nabla^{\prime 2} \boldsymbol{u}^{\prime}+\operatorname{PrRa}\left(1-z^{\prime}+\theta^{\prime}\right) \boldsymbol{e}_{z}=\lambda \boldsymbol{f}^{\prime}, \\
-\left(\boldsymbol{u}^{\prime} \cdot \nabla^{\prime}\right) \theta^{\prime}+w^{\prime}+\nabla^{\prime 2} \theta^{\prime}=0, \\
\nabla^{\prime} \cdot \boldsymbol{u}^{\prime}=0,
\end{gathered}
$$

where $\lambda$ is a homotopy parameter. For fixed $\operatorname{Ra}=10^{4}, \operatorname{Pr}=1$ and $f^{\prime}$, we have tracked the solution from $\lambda=1$ to 0 using the Newton-Krylov method (figure 8). The connected solution $S_{3 D}$ is the present 3-D steady solution shown in $\S \S 3$ and 4. 


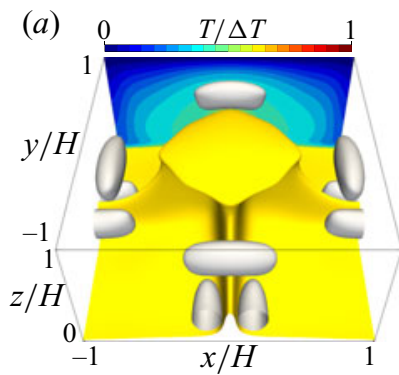

(b)

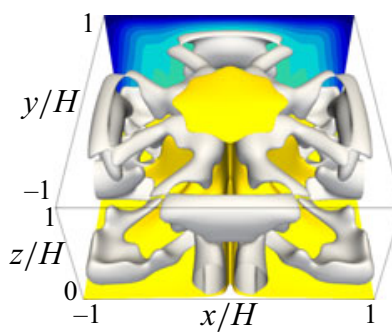

(c)

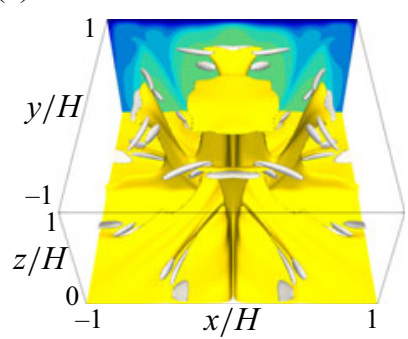

Figure 10. The 3-D steady solution in the domain with $L / H=2 \pi / 3.117 \approx 2.02$ for $\operatorname{Pr}=1$ at $(a) \operatorname{Ra}=10^{5}$, (b) $R a=10^{6}$ and (c) $R a=10^{7}$. The yellow and grey objects represent the isosurfaces of $T / \Delta T=0.6$ and (a) $Q /\left(\kappa^{2} / H^{4}\right)=1.28 \times 10^{5}$, (b) $Q /\left(\kappa^{2} / H^{4}\right)=8 \times 10^{5}$ and (c) $Q /\left(\kappa^{2} / H^{4}\right)=8 \times 10^{7}$, respectively. The contours indicate $T$ in the plane $y / H=\pi / 3.117$.
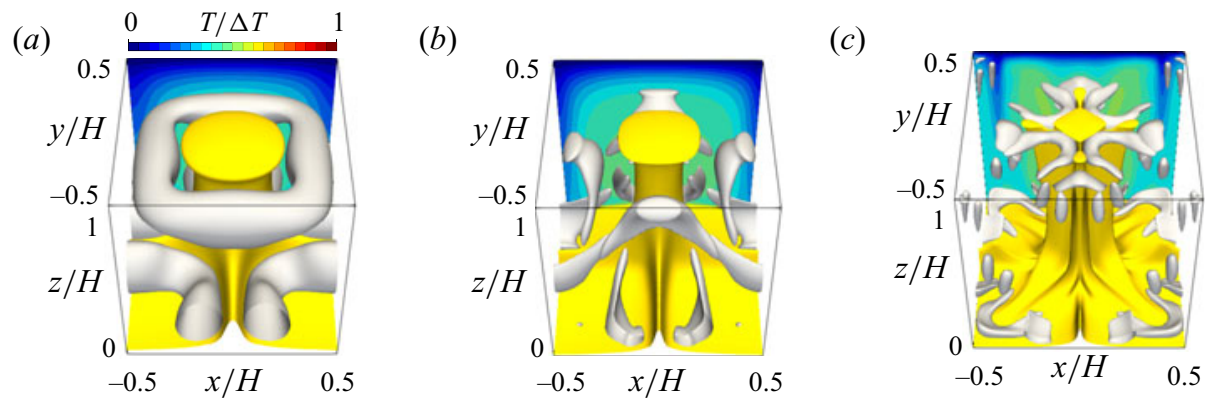

Figure 11. The 3-D steady solution in the domain with $L / H=1$ for $\operatorname{Pr}=1$ at (a) $R a=10^{5}$, (b) $R a=10^{6}$ and (c) $R a=10^{7}$. The yellow and grey objects represent the isosurfaces of $T / \Delta T=0.6$ and $(a) Q /\left(\kappa^{2} / H^{4}\right)=$ $1.28 \times 10^{5},(b) Q /\left(\kappa^{2} / H^{4}\right)=2.4 \times 10^{6}$ and $(c) Q /\left(\kappa^{2} / H^{4}\right)=5 \times 10^{7}$, respectively. The contours indicate $T$ in the plane $y / H=0.5$.
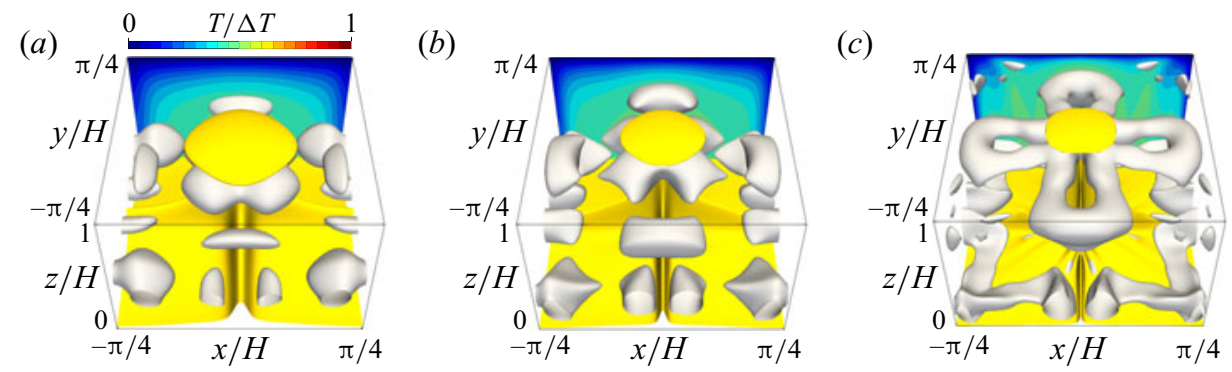

Figure 12. The 3-D steady solution in the domain with $L / H=\pi / 2 \approx 1.57$ for $\operatorname{Pr}=7$ at (a) $\operatorname{Ra}=10^{5}$, (b) $R a=10^{6}$ and (c) $R a=10^{7}$. The yellow and grey objects represent the isosurfaces of $T / \Delta T=0.6$ and (a) $Q /\left(\kappa^{2} / H^{4}\right)=1 \times 10^{5}$, (b) $Q /\left(\kappa^{2} / H^{4}\right)=1 \times 10^{6}$ and (c) $Q /\left(\kappa^{2} / H^{4}\right)=1.28 \times 10^{7}$, respectively. The contours indicate $T$ in the plane $y / H=\pi / 2$.

\section{Appendix B. Dependence of multi-scale steady solution on domain size, Prandtl number and spatial resolution}

Figure 9 shows the Nusselt number $\mathrm{Nu}$ compensated by $R a^{0.31}$ and the Reynolds number $R e$ compensated by $\mathrm{Pr}^{-2 / 3} \mathrm{Ra}^{4 / 9}$ as a function of the Rayleigh number $R a$ in the 3-D steady solutions for different horizontal period $L$ and Prandtl number $P r$. The magenta, red and 


$\begin{array}{lcccccccc} & R a & \left(N_{x}, N_{y}, N_{z}\right) & \begin{array}{c}\Delta x / H \\ \times 10^{-2}\end{array} & \begin{array}{c}\Delta z / H \\ \times 10^{-2}\end{array} & \begin{array}{c}\langle\eta\rangle_{z} / H \\ \times 10^{-2}\end{array} & \begin{array}{c}\left.\eta\right|_{c} / H \\ \times 10^{-2}\end{array} & \begin{array}{c}\delta / H \\ \times 10^{-2}\end{array} & \tau /(U / H) \\ & & & & & & & & \\ \text { 3-D steady } & 10^{5} & (128,128,128) & 1.22 & 0.0153-1.24 & 3.92 & 4.25 & 9.57 & - \\ \text { solution } & 10^{6} & (128,128,128) & 1.22 & 0.0153-1.24 & 1.82 & 2.08 & 4.95 & - \\ & 10^{7} & (256,256,256) & 0.611 & 0.00379-0.616 & 0.859 & 1.01 & 2.59 & - \\ & 10^{7.42} & (256,256,256) & 0.611 & 0.00379-0.616 & 0.638 & 0.746 & 2.08 & - \\ \text { Turbulent } & 10^{5} & (128,128,128) & 1.22 & 0.0153-1.24 & 3.99 & 4.85 & 10.1 & 7906 \\ \text { states } & 10^{6} & (128,128,128) & 1.22 & 0.0153-1.24 & 1.88 & 2.05 & 5.57 & 2500 \\ & 10^{7} & (256,256,256) & 0.611 & 0.00379-0.616 & 0.897 & 0.945 & 3.04 & 1028 \\ & 10^{7.42} & (256,256,256) & 0.611 & 0.00379-0.616 & 0.657 & 0.685 & 2.34 & 513\end{array}$

Table 1. Numerical details of the 3-D steady solution and the turbulent states for $L / H=\pi / 2$ and $\operatorname{Pr}=1$. Here $\Delta x$ and $\Delta z$ are the spatial resolutions in the $x$ and $z$ directions; $\langle\eta\rangle_{z}$ and $\left.\eta\right|_{c}$ represent the Kolmogorov micro-scale length $\eta=\left(v^{3} / \varepsilon\right)^{1 / 4}$ based on the vertical averaged energy dissipation rate, $\langle\varepsilon\rangle_{z}=$ $(v / 2)\left\langle\left(\partial u_{i} / \partial x_{j}+\partial u_{j} / \partial x_{i}\right)^{2}\right\rangle_{x y z t}$, and that at the centre of the fluid layer, $\left.\varepsilon\right|_{z=H / 2}$, respectively; $\delta$ is the thermal conduction layer thickness, $\delta / H=1 /(2 N u) ; \tau$ is the integral time to obtain the statistics; and $U=(g \alpha \Delta T H)^{1 / 2}$ is the buoyancy-induced terminal velocity.

orange symbols represent $L / H=2 \pi /\left(k_{c} H\right) \approx 2.02, \pi / 2 \approx 1.57$ and 1 , respectively, for $\operatorname{Pr}=1$, where $k_{c}=3.117 / H$ is the wavenumber corresponding to the minimal critical Rayleigh number $R a_{c}=1708$ (Drazin \& Reid 1981). Although there is a variation for small $R a$, the three solutions show similar $N u$ at $R a \gtrsim 10^{6}$. The green symbols represent $L / H=\pi / 2$ for $\mathrm{Pr}=7$, and the dependence of $\mathrm{Nu}$ on $\mathrm{Pr}$ is minor. The dependence of $\mathrm{Nu}$ on the domain size and $\operatorname{Pr}$ (for $1 \lesssim \operatorname{Pr} \lesssim 10$ ) is expected to not be significant at a high $R a$, since the emergence of the small-scale plume and vortex structures near the walls, which are robustly observed for different values of $L$ and $\operatorname{Pr}$ (figures 5, 10, 11 and 12), might be a key ingredient in the vertical heat flux. In contrast, a variation in $R e$ exists; however, all the solutions are comparable at $R a \sim 10^{7}$. The blue filled and open diamonds in figure 9(a) indicate the 2-D optimised steady solutions for $\operatorname{Pr}=1$ and 7, respectively, obtained by Sondak et al. (2015). The 3-D steady solutions can exceed the maximal heat flux in the 2-D steady solutions. Meanwhile, at $R a \gtrsim 10^{6}$ the present 3-D steady solutions with $1 \leq L / H \lesssim 2$ exhibit smaller $N u$ than those of the 2 -D optimised steady solutions at the same $\mathrm{Pr}$.

In figure 9 the symbols $+, \bullet, \times$ and $\circ$ show the results obtained on different grid points $\left(N_{x}, N_{y}, N_{z}\right)=(64,64,64),(128,128,128),(192,192,128)$ and $(256,256,256)$, respectively, and the effects of the spatial resolutions on $N u$ and $R e$ are minor. For our main results with $L / H=\pi / 2$ and $\operatorname{Pr}=1$, the grid points $\left(N_{x}, N_{y}, N_{z}\right)=(128,128,128)$ are enough to evaluate the characteristics of the 3 -D steady solution at $R a \lesssim 10^{7}$; $\left(N_{x}, N_{y}, N_{z}\right)=(256,256,256)$ are sufficient at $R a \sim 10^{7}$. Furthermore, the Kolmogorov micro-scale length $\eta$ and the thermal conduction layer thickness $\delta$ in the 3-D steady solution and the turbulent states at $R a=10^{5}, 10^{6}, 10^{7}$ and $10^{7.42} \approx 2.6 \times 10^{7}$ are summarised in table 1 together with the grid sizes. Since the energy dissipation rate is a function of the wall-normal coordinate $z, \varepsilon(z)=(v / 2)\left\langle\left(\partial u_{i} / \partial x_{j}+\partial u_{j} / \partial x_{i}\right)^{2}\right\rangle_{x y t}, \eta$ also depends on $z$. Here $\langle\eta\rangle_{z}$ and $\left.\eta\right|_{c}$ are based on the total energy dissipation rate, $\langle\varepsilon\rangle_{z}$, and that at the centre of the fluid layer, $\left.\varepsilon\right|_{z=H / 2}$, respectively, and $\langle\eta\rangle_{z}<\left.\eta\right|_{c}$ in all cases. The grid size in the $x$ direction, $\Delta x(=\Delta y)$, and the maximal value of $z, \Delta z$, are comparable with $\eta$ and less than one-third of $\delta$. Therefore, the spatial resolution is sufficient to resolve 


\section{Multi-scale steady solution for Rayleigh-Bénard convection}

the smallest-scale thermal and flow structures in the 3-D steady solution and the turbulent states. The present turbulent direct numerical simulation data are obtained by averaging time of more than 200 convective time units based on the buoyancy-induced terminal velocity $U=(g \alpha \Delta T H)^{1 / 2}$.

\section{REFERENCES}

Ahlers, G., Grossmann, S. \& Lohse, D. 2009 Heat transfer and large scale dynamics in turbulent Rayleigh-Bénard convection. Rev. Mod. Phys. 81, 503-537.

BATCHELOR, G.K. 1961 Considerations of convective instability from the viewpoint of physics - Discussion. In Aerodynamic Phenomena in Stellar Atmospheres, Proceedings of the 4th Symposium on Cosmical Gas Dynamics, August 18-30, 1960 (ed. R.N. Thomas), pp. 385-402. N. Zanichelli.

BusSE, F.H. 1969 On Howard's upper bound for heat transport by turbulent convection. J. Fluid Mech. 37, 457-477.

Castaing, B., Gunaratne, G., Heslot, F., Kadanoff, L., Libchaber, A., Thomae, S., Wu, X.Z., ZALESKI, S. \& ZANETTI, G. 1989 Scaling of hard thermal turbulence in Rayleigh-Bénard convection. J. Fluid Mech. 204, 1-30.

Chavanne, X., Chilla, F., Castaing, B., Hebral, B., Chabaud, B. \& Chaussy, J. 1997 Observation of the ultimate regime in Rayleigh-Bénard convection. Phys. Rev. Lett. 79, 3648-3651.

Chillà, F. \& Schumacher, J. 2012 New perspectives in turbulent Rayleigh-Bénard convection. Eur. Phys. J. E 35, 58.

DoERING, C.R. 2019 Thermal forcing and 'classical' and 'ultimate' regimes of Rayleigh-Bénard convection. J. Fluid Mech. 868, 1-4.

Doering, C.R. \& Constantin, P. 1992 Energy dissipation in shear driven turbulence. Phys. Rev. Lett. 69, $1648-1651$.

Doering, C.R. \& Constantin, P. 1996 Variational bounds on energy dissipation in incompressible flows. I. Convection. Phys. Rev. E 53 (6), 5957-5981.

Doering, C.R. \& Tobasco, I. 2019 On the optimal design of wall-to-wall heat transport. Commun. Pure Appl. Maths 72, 2385-2448.

Doering, C.R., Toppaladoddi, S. \& Wettlaufer, J.S. 2019a Absence of evidence for the ultimate regime in two-dimensional Rayleigh-Bénard convection. Phys. Rev. Lett. 123, 259401.

Doering, C.R., Toppaladoddi, S. \& Wettlaufer, J.S. $2019 b$ Absence of evidence for the ultimate regime in two-dimensional Rayleigh-Bénard convection. arXiv:1912.07698.

DRAZIN, P.G. \& REID, W.H. 1981 Hydrodynamic Stability. Cambridge University Press.

Goto, S., Saito, Y. \& Kawahara, G. 2017 Hierarchy of antiparallel vortex tubes in spatially periodic turbulence at high Reynolds numbers. Phys. Rev. Fluids 2, 064603.

Grossmann, S. \& LohSE, D. 2000 Scaling in thermal convection: a unifying theory. J. Fluid Mech. 407, $27-56$.

Grossmann, S. \& Lohse, D. 2001 Thermal convection for large Prandtl numbers. Phys. Rev. Lett. 86 (15), 3316-3319.

Grossmann, S. \& Lohse, D. 2002 Prandtl and Rayleigh number dependence of the Reynolds number in turbulent thermal convection. Phys. Rev. E 66, 016305.

Grossmann, S. \& LohSE, D. 2004 Fluctuations in turbulent Rayleigh-Bénard convection: the role of plumes. Phys. Fluids 16 (012), 4462-4472.

Grossmann, S. \& Lohse, D. 2011 Multiple scaling in the ultimate regime of thermal convection. Phys. Fluids 23, 045108.

Hassanzadeh, P., Chini, G.P. \& Doering, C.R. 2014 Wall to wall optimal transport. J. Fluid Mech. 751, $627-662$.

He, X., Funfschilling, D., Nobach, H., Bodenschatz, E. \& Ahlers, G. 2012 Transition to the ultimate state of turbulent Rayleigh-Bénard convection. Phys. Rev. Lett. 108, 024502.

HowARD, L.N. 1963 Heat transport by turbulent convection. J. Fluid Mech. 17, 405-432.

Howard, L.N. 1966 Convection at high Rayleigh number. In Applied Mechanics, Proceedings of the Eleventh International Congress of Applied Mechanics (ed. H. Görtler), pp. 1109-1115. Springer.

Ishihara, T., Morishita, K., Yokokawa, M., Uno, A. \& Kaneda, Y. 2016 Energy spectrum in high-resolution direct numerical simulations of turbulence. Phys. Rev. Fluids 1, 082403(R).

IYer, K.P., Scheel, J.D., Schumacher, J. \& Sreenivasan, K.R. 2020 Classical 1/3 scaling of convection holds up to Ra $=10^{15}$. Proc. Natl Acad. Sci. USA 117, 7594. 


\section{S. Motoki, G. Kawahara and M. Shimizu}

Kawano, K., Motoki, S., Shimizu, M. \& Kawahara, G. 2021 Ultimate heat transfer in 'wall-bounded' convective turbulence. J. Fluid Mech. 914, A13.

Kolmogorov, A.N. 1941 The local structure of turbulence in incompressible viscous fluid for very large Reynolds numbers. Dokl. Akad. Nauk SSSR 30, 301-305.

KRAICHNAN, R.H. 1962 Turbulent thermal convection at arbitrary Prandtl number. Phys. Fluids 5, 1374.

Lozano-Durán, A., Holzner, M. \& Jimenez, J. 2016 Multiscale analysis of the topological invariants in the logarithmic region of turbulent channels at a friction Reynolds number of 932. J. Fluid Mech. 803, 356-394.

Malkus, W.V.R. 1954 The heat transport and spectrum of thermal turbulence. Proc. R. Soc. Lond. A 225, $196-212$

MizunO, Y. 2016 Spectra of energy transport in turbulent channel flows for moderate Reynolds numbers. J. Fluid Mech. 805, 171-187.

Motoki, S., Kawahara, G. \& ShimizU, M. $2018 a$ Maximal heat transfer between two parallel plates. J. Fluid Mech. 851, R4.

Motoki, S., Kawahara, G. \& Shimizu, M. $2018 b$ Optimal heat transfer enhancement in plane Couette flow. J. Fluid Mech. 835, 1157-1198.

Motoori, Y. \& Goto, S. 2019 Generation mechanism of a hierarchy of vortices in a turbulent boundary layer. J. Fluid Mech. 865, 1085-1109.

Niemela, J. \& SREEnivasAn, K.R. 2006 Turbulent convection at high Rayleigh numbers and aspect ratio 4. J. Fluid Mech. 557, 411-422.

Niemela, J.J., Skrbek, L., Sreenivasan, K.R. \& Donnelly, R.J. 2000 Turbulent convection at very high Rayleigh numbers. Nature 404, 837-840.

Plasting, S.C. \& Kerswell, R.R. 2003 Improved upper bound on the energy dissipation rate in plane Couette flow: the full solution to Busse's problem and the Constantin-Doering-Hopf problem with one-dimensional background field. J. Fluid Mech. 477, 363-379.

Priestley, C.H.B. 1954 Convection from a large horizontal surface. Aust. J. Phys. 7, 176-201.

Shraiman, B.I. \& Siggia, E.D. 1990 Heat transport in high-Rayleigh-number convection. Phys. Rev. A 42 (6), 3650-3653.

Silano, G., Sreenivasan, K.R. \& VerzicCO, R. 2010 Numerical simulations of Rayleigh-Bénard convection for Prandtl numbers between $10^{-1}$ and $10^{4}$ and Rayleigh numbers between $10^{5}$ and $10^{9}$. J. Fluid Mech. 662, 409-446.

Sondak, D., Smith, L.M. \& WalefFe, F. 2015 Optimal heat transport solutions for Rayleigh-Bénard convection. J. Fluid Mech. 784, 565-595.

SouzA, A.N., Tobasco, I. \& Doering, C.R. 2020 Wall-to-wall optimal transport in two dimensions. J. Fluid Mech. 889, A34.

SpIEGEL, E.A. 1963 A generalization of the mixing-length theory of thermal convection. Astrophys. J. 138, 216-225.

SReEnivaSAn, K.R. 1995 On the universality of the Kolmogorov constant. Phys. Fluids 7, 2778.

Stevens, R.J.A.M., VAn Der Poel, E.P., Grossmann, S. \& Lohse, D. 2013 The unifying theory of scaling in thermal convection: the updated prefactors. J. Fluid Mech. 730, 295-308.

Tobasco, I. \& Doering, C.R. 2017 Optimal wall-to-wall transport by incompressible flows. Phys. Rev. Lett. 118, 264502.

van Veen, L., Vela-Martín, A. \& Kawahara, G. 2019 Time-periodic inertial range dynamics. Phys. Rev. Lett. 123, 134502.

Waleffe, F., Boonkasame, A. \& Smith, L.M. 2015 Heat transport by coherent Rayleigh-Bénard convection. Phys. Fluids 27, 051702.

Zhu, X., Mathai, V., Stevens, R.J.A.M., Verzicco, R. \& Lohse, D. 2018 Transition to the ultimate regime in two-dimensional Rayleigh-Bénard convection. Phys. Rev. Lett. 120, 144502. 